\title{
Vision « industrielle » de la gestion de l'eau sur un périmètre irrigué
}

\author{
par Jacques Rey \\ Cemagref Montpellier \\ Daniel Renault \\ IIMI (Sri-Lanka) \\ Sophie Lamacq \\ Cemagref Montpellier
}

\section{INTRODUCTION}

Il est proposé dans cet article d'aborder la gestion de l'eau dans les périmètres irrigués sous l'angle de la «gestion de production ». Ce domaine d'investigation, très classique en situation d'entreprise industrielle, a en effet suscité la mise au point d'un certain nombre de méthodes et d'outils d'intervention, susceptibles de faire avancer la réflexion sur la gestion des périmètres irrigués. La question plus spécifique de la participation des agriculteurs à la gestion de l'eau et de leurs relations avec une agence d'irrigation s'inscrira dès lors dans la problématique de la coordination et du pilotage d'un processus de production où interviennent plusieurs acteurs. Pour mener à bien une analyse de ce type, on précisera dans un premier temps les principaux termes d'une analogie entre systèmes de production industriels et périmètres irrigués. Cette analogie permettra ensuite d'établir un mode de représentation du système de pilotage de la production « d'eau maîtrisée » à l'échelle du périmètre. A l'aide de cette représentation il sera enfin possible d'esquisser une démarche d'intervention, prenant en compte les problèmes

\section{Can irrigation management learn something from industry?}

Inproning the performance of irrigation systems by promoting better mungenent is a fashionable subject. Unfortunatety. the definitions of $r$ perfomances and t management are among the most controvervial in the irigation communin. This falime 10 grasp the the meaning of such crucial terms has often led to misunderstandings and confuston. This article intends to bring the debates in a inore neutrat and raisona. bly well understood territory. the field of industrial production swstems. The hypothesis underlying this approach is that the terms of an analogy between the t prodtction process $\lambda$ of water in an imgation sys. tern and a standard indisinal process can be made more precise. Moreoner the specific objective of the article is to defime the basics of a nethod of intervention for analysis and improvement of water management performance in irigailon systems inspired by the expertences and concepts of industrial nanagement. 
de coordination de production, pour améliorer la gestion de l'eau dans les périmètres irrigués.

\section{ANALOGIE PÉRIMÈTRE IRRIGUÉ / ENTREPRISE INDUSTRIELLE}

Dans cet article, le système «périmètre irrigué » est défini comme une "communauté d'acteurs, avec tous leurs moyens de production, qui sont rendus interdépendants par la production etlou l'utilisation partagée « d'eau mâ̂trisée » dans le but de produire des biens agricoles ». Cette définition permet de relier clairement la notion de périmètre irrigué à l'identification d'une ressource en eau « partagée ».

Pour aborder le sujet choisi de façon opérationnelle et structurer en conséquence l'analogie périmètre/entreprise, on se limitera à une perspective «système de production" (ignorant en cela les volets financiers, commerciaux et administratifs de l'entreprise).

\subsection{Systèmes de production, interface}

Le système de production principal d'un périmètre irrigué est évidemment le système de production de biens agricoles à l'échelle de ce périmètre. Une vision purement mécaniste du fonctionnement de ce système de production amène à considérer un faisceau de processus de production et/ou de distribution d'intrants (tels que l'eau, les engrais...), devant être « orchestré » pour un assemblage final au sein d'ateliers avals (les parcelles), produisant les biens agricoles.

L'objet de l'article est d'étudier la production d'un intrant particulier: l'eau qualifiée de maîtrisée. Le processus de production de cet intrant couvre l'ensemble des transformations effectuées sur l'eau depuis la source alimentant le périmètre jusqu'à l'application sur les parcelles. Le mode d'organisation adopté sur les périmètres conduit dans la plupart des cas à mettre en évidence une interface de gestion entre l'exploitation agricole et l'agence d'irrigation au sein d'un périmètre irrigué. A cette interface, l'acteur en charge de l'eau maitrisée passe d'une logique de producteur à une logique d'utilisateur de cet intrant. L'interface de gestion se situe souvent à un niveau donné de ramification du réseau hydraulique, par exemple au niveau tertiaire.

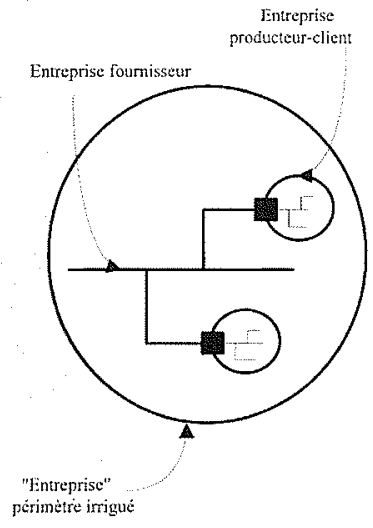

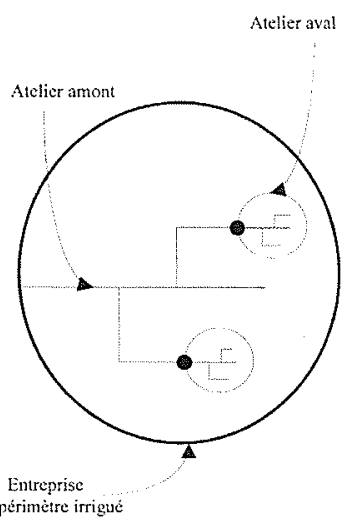

Legende

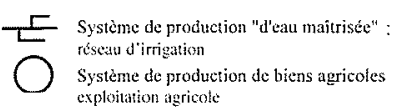

Interface d'entreprises

Interface d'ateliers

1. Deux types d'interfaces possibles entre l'exploitation agricole et l'agence d'irrigation au sein d'un périmètre irrigué.

De façon traditionnelle sur les grands périmètres, l'interface met en présence une agence d'irrigation (acteur "producteur » d'eau maîtrisée) et les agriculteurs, individuels ou regroupés en association (acteur «utilisateur» d'eau maîtrisée). Cette configuration s'apparente en industrie au cas où une entreprise amont (fournisseur) traite avec de multiples entreprises aval (producteurs-clients). Sur l'essentiel des petits périmètres néanmoins, l'acteur producteur est soi agriculteur soit directement mandaté par les agriculteurs; la configuration de gestion plus intégrée qui en résulte s'apparente alors plutôt à un cas d'entreprise unique avec un atelier amont fournissant de multiples ateliers aval (figure 1).

\subsection{Le produit « eau maîtrisée 》}

La poursuite de l'analogie implique de définir plus précisément le produit «eau maîtrisée», objet de la transaction à l'interface de gestion. Selon une terminologie d'entreprise on peut identifier un produit industriel : I'hydrogramme ou profil de débit au cours du temps (figure 2).

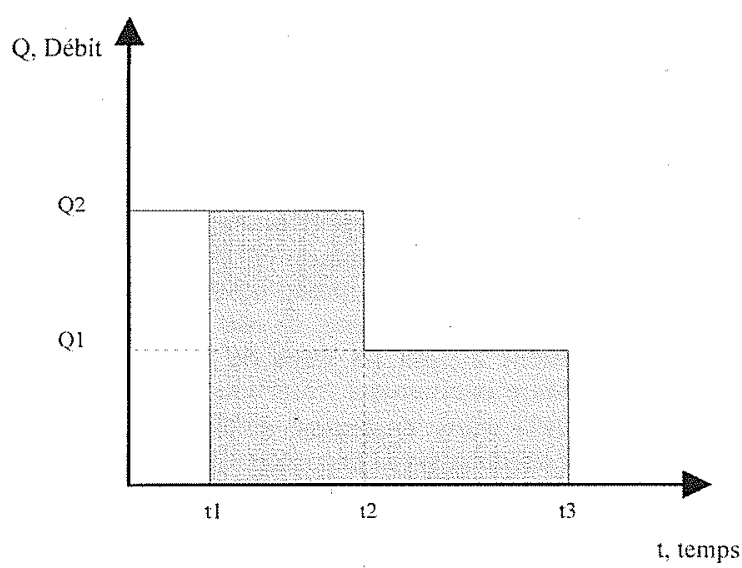

2. Un hydrogramme.

Ce produit est fourni par un producteur spécialisé, dans des conditions de service variables (catalogue plus ou moins important, latitude dans le choix des moments de livraison) qui dépendent des effets combinés de contraintes techniques de production et des choix de gestion régissant les relations entre les deux acteurs de l'interface (le gestionnaire du réseau d'irrigation et l'exploitant agricole).

\subsection{Machines, processus}

Le processus de fabrication du produit «eau maîtrisée » est non trivial. Sur le segment amont du processus, la matière première est transformée en produit semi-fini non différentié à l'aide d'ouvrages hydrauliques spécialisés (« machines » telles que des vannes, seuils, pompes...) ; une ultime opération sur des machines situées à l'interface de gestion assure de façon simultanée la différentiation du produit semi-fini et la livraison à chaque «producteur-client». Le segment aval se situe chez les «producteurs-clients» et permet d'effectuer les dernières opérations transformant le produit livré "eau maîtrisée » en produit final, utilisé comme intrant dans un itinéraire cultural (au niveau des parcelles). 
Les lois régissant la fabrication sont essentiellement celles de l'hydraulique, parfois sous pression (notamment sur le deuxième segment), le plus souvent à surface libre (presqu'exclusivement sur le premier segment). La nature des équations sous-jacentes (non-linéarité, retard) fait que les gammes de production sont difficiles à optimiser en fonction des trois objectifs classiques de performance : «coût » (coût des moyens mis en wuvre et des approvisionnements en matière première), " délais » (délais de réponse à des besoins exprimés et temps de cycle) et «qualité » (écart entre produits livrés et produits attendus).

\section{粮 2.4 Logique de pilotage}

En fonction de la logique de pilotage choisie, un système de production s'oriente vers davantage de réactivité face à des demandes aval exprimées (production en "flux tirés») ou davantage de planification basée sur des prévisions de cette même demande aval (production en «flux poussés»). Une logique de pilotage est bien évidemment déployée par l'intermédiaire des décisions que prennent les différents pilotes en charge du processus. En ce qui concerne le processus de production d'eau maîtrisée, on peut retenir trois grandes classes de décisions de pilotage:

1. L'allocation saisonnière de la ressource (AS), 2. La programmation intra-saisonnière des irrigations (PI), 3. Les plans de mancuvre des ouvrages hydrauliques (PM). - La décision (AS) relève de la planification de production et consiste à raisonner en début de saison, en fonction des disponibilités potentielles d'approvisionnement en eau, les surfaces pouvant être mises en culture (et éventuellement les types de culture). Cette décision prend toute son importance dans des périmètres où la ressource en eau est limitée et où on est parfois amené à gérer un stock de matière première inter-saisonnier voire inter-annuel (réservoir).

- La décision (PI) relève également de la planification de production et consiste à fixer des objectifs de production d'eau maitrisée à un pas de temps plus fin et de façon plus précise ; elle touche donc également à l'ordonnancement dans la mesure où programmer une livraison revient à programmer la dernière opération d'une gamme de production. - La décision (PM) relève de l'ordonnancement au sens strict; il s'agit de préciser la gamme de production en raisonnant la succession des opérations à effectuer sur les ouvrages hydrauliques pour mener à bien la fabrication des produits devant être livrés. Les flux de matière sont régulés en fonction de prévisions centralisées des besoins (commandes en «boucle ouverte ») et en fonction de mesures locales d'écarts de niveaux d'eau ou de débit par rapport à des situations de consigne (commandes en «boucle fermée »). Les deux types d'approche se retrouvent en industrie où l'on utilise assez largement des outils de type flux poussé comme la GPAO $\left(^{1}\right)$ pour instrumenter l'élaboration des commandes en boucle ouverte et des outils de type flux tirés comme les KANBAN $\left({ }^{2}\right)$ pour instrumenter l'élaboration des commandes en boucle fermée.

Que conclure de ce bref parallèle entre périmètres irrigués et systèmes de production industriels ? La création de valeur à l'échelle d'un périmètre dépend $\mathrm{du}$ « bon » fonctionnement du système de production de biens agricoles à cette échelle. Dans ce demier est inclus un système principal de production d'eau maîtrisée, fournissant de nombreux sous-systèmes

(1) Gestion de Production Assistée par Ordinateur

(2) Méthode de gestion d'origine Japonaise permettant la transmission de demandes locales de l'aval vers l'amont entre les postes de travail pour déclencher les opérations de production. de production de biens agricoles par l'intermédiaire d'une interface de gestion. Cette interface coupe le processus de production d'eau mâtrisée et est donc susceptible de poser des problèmes en matière de coordination de production. Cette dernière remarque conduit à analyser de façon globale et transversale le pilotage de ce processus.

\section{I REPRÉSENTATION DU SYSTÈME DE PILOTAGE}

\subsection{Architecture}

L'essentiel des avancées réalisées en industrie sur les problèmes de représentation ont été inspirées par la remise en cause des systèmes traditionnels de contrôle de gestion. L'affectation analytique des coûts et les mesures de performance associées ne se satisfont plus du cadre comptable classique et poussent à l'élaboration de représentations qui collent de plus près à la réalité de la production et de la création de valeur dans l'entreprise. On peut retenir deux concepts principaux issus de ces nouvelles lectures de la production : le concept d'activité [1] et le concept de processus [2]. Toute création de valeur par un système de production est associée à une résultante de ce système créée au sein d'un processus constitué d'une succession d'activités de pilotage élémentaires.

En ce qui concerne la production d'eau maitrisée, il est donc naturel de chercher à situer les activités de pilotage traitant des trois grandes décisions évoquées précédemment (niveaux AS, PI et PM) au sein de processus créateurs de valeur sur le périmètre irrigué. Cela conduit à mettre en évidence deux types de relations fondamentalement différentes entre les pilotes (groupes de personnes - auquel cas la décision est produite par l'interaction de plusieurs cerveaux via négociation ou autre - algorithme...) :

1. Des relations hiérarchiques de type pilote/piloté entre pilotes de même niveau ou de niveaux différents (dans ce dernier cas : relations AS/PI et PI/PM).

2. Des relations non hiérarchiques de type pilotelpilote ou coordination de processus entre pilotes d'un même niveau ; les pilotes de niveau PM interviennent sur le processus de production d'eau maîtrisée, ceux de niveau PI sur le processus de production de biens agricoles et ceux de niveau AS sur le processus de production de richesses à l'échelle du périmètre.

La figure 3 permet d'illustrer les notions précédentes en utilisant un système hypothétique sur lequel auraient été identifiés treize pilotes.

Cette représentation permet ainsi de dresser une carte sommaire des relations entre les divers pilotes impliqués dans la gestion de l'eau sur un périmètre irrigué : agence d'irrigation, association d'agriculteurs, prestataire de service privé... et de situer leurs rôles respectifs à la fois dans la hiérarchie de pilotage et dans les processus créateurs de valeur. Elle peut être notablement précisée en faisant appel à des techniques d'analyse utilisées dans l'industrie (par exemple, en France, l'Analyse Modulaire des Systèmes [3]) pour formaliser plus finement certains aspects génériques des relations de pilotage.

\subsection{Instrumentation}

Une représentation sommaire du type précédent prend toute sa signification lorsque l'on essaye d'expliciter concrètement la «mécanique " de fonctionnement qui s'établit entre les divers pilotes d'un périmètre particulier. La mise à plat de cette mécanique conduit à «animer » la représentation en y projetant de manière désormais structurée le détail des com- 


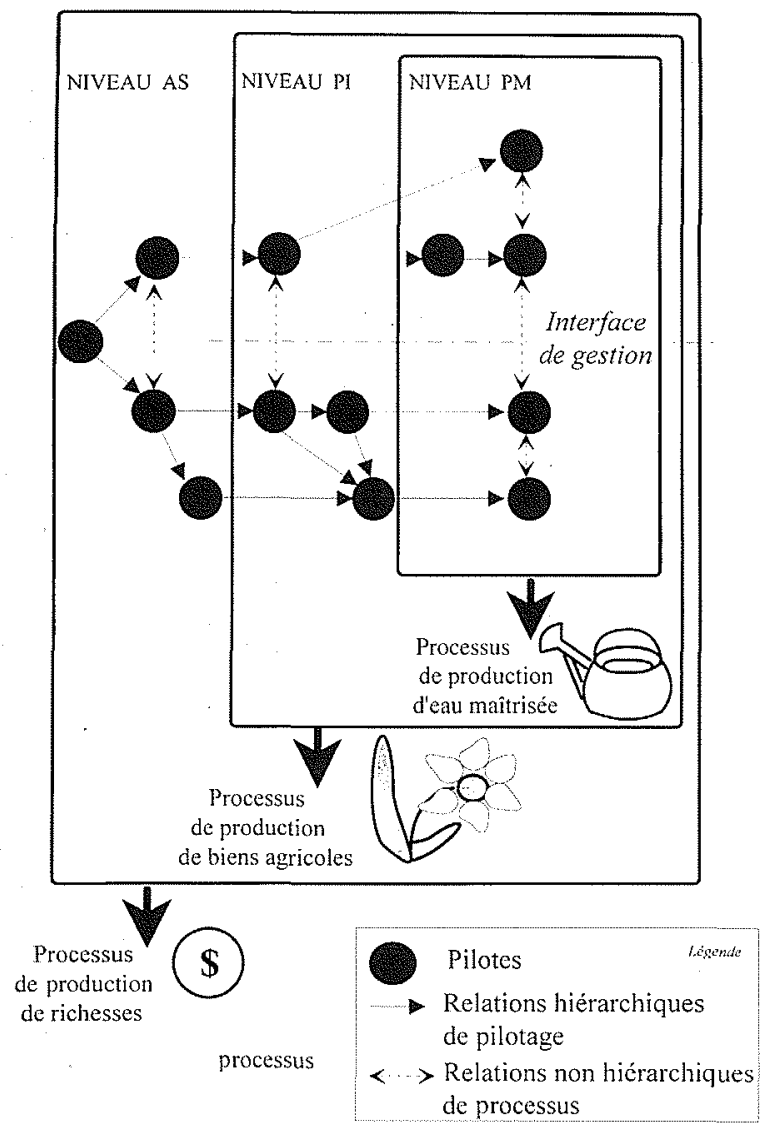

3. Pilotage et processus créateurs de valeur sur un périmètre irrigué.

munications échangées par les pilotes, des traitements qu'ils effectuent et des données qu'ils manipulent (messages, logiciels, tableaux de bord...). Cette étape peut être réalisée dans une perspective classique d'analyse d'un système d'information en faisant appel, là encore, à des techniques éprouvées (par exemple, en France, Mérise [4]). On notera que les conclusions du premier paragraphe incitent à accorder une attention toute particulière à l'instrumentation, aux relations non hiérarchiques de processus entre pilotes de même niveau (quelles communications échangées, quelle influence sur les traitements, quelles données stockées ?).

L'explicitation d'une représentation formalisée du système de pilotage de la production d'eau mâitrisée et surtout de l'instrumentation qui y est associée permet donc d'analyser finement la chaîne de pilotage hiérarchique et l'interface de gestion production/utilisation d'eau maîtrisée. On va montrer dans la suite que cette représentation constitue en fait un point d'ancrage naturel pour toute intervention visant à améliorer la performance de la gestion de l'eau sur un périmètre irrigué. On inscrira ensuite cette phase liminaire d'explicitation dans une « trame » complète d'intervention.

\section{4. 圆 DÉMARCHE D'AMÉLIORATION DE LA GESTION DE L'EAU DANS UN PÉRIMÈTRE IRRIGUÉ}

\subsection{Un point d'ancrage « performance»}

On adopte ici résolument le point de vue d'un intervenant mandaté pour analyser la performance de la gestion de l'eau dans un périmètre et faire des propositions d'action pour l'améliorer. Parler de la performance d'un système de production, quel qu'il soit, impose de raisonner en terme d'acteurs. On est amené à distinguer les acteurs «clients" du système de production de l'acteur «gestionnaire»; les premiers sanctionnent la «valeur». (à leurs yeux) des activités du système, le second doit anticiper ces «interprétations valeur », les formuler en terme d'objectifs stratégiques puis les déployer dans son système de production à travers une «interprétation performance». Un système n'est performant qu'au vu de la réalisation d'un objectif stratégique, donc au vu de la vision d'un acteur client, interprétée par un acteur gestionnaire. En matière de gestion de l'eau sur un périmètre, on peut identifier différents niveaux d'acteurs clients correspondant aux trois processus principaux de création de valeur mis en évidence précédemment.

- Processus de production d'eau maitrisée: dans une configuration de type fournisseur/producteur-client, on a effectivement une classe d'acteurs clients du système de production d'eau maîtrisée (interprétation valeur en terme de qualité totale du produit eau maîtrisée). Dans une configuration atelier/atelier, on ne retrouve pas cette notion (les vrais clients seraient ici les cultures irriguées...).

- Processus de production de biens agricoles: les acteurs clients sont évidemment les marchés agricoles (interprétation valeur en terme de qualité totale des produits agricoles). - Processus de création de richesses à l'échelle du périmètre : les acteurs clients peuvent à ce niveau avoir des visages divers; on retiendra les actionnaires du périmètre (interprétation valeur en terme de rentabilité) et les pouvoirs réglementaires (interprétation valeur en terme de justice sociale, de durabilité, de productivité des ressources rares...).

On conçoit aisément que les acteurs gestionnaires des niveaux AS, PI et PM aient parfois du mal à déployer l'ensemble des objectifs stratégiques (parfois contradictoires) qui découlent de l'analyse précédente, que se soit au sein d'une hiérarchie de pilotage ou à travers la coordination des processus. Il n'est en fait pas réaliste d'envisager un tel déploiement sans un système de pilotage cohérent, transparent et bien instrumenté.

On peut mentionner dès à présent que les expériences conduites en milieu industriel pour améliorer la production incitent à être encore plus catégoriques: pour intervenir de façon pertinente et durable, il faut disposer d'une représentation du système de pilotage comprise et partagée par les différents acteurs [5], [6].

\subsection{Une trame opératoire}

Le temps de l'action est généralement court pour un intervenant. Juger de la «fonctionnalité » d'un système sans avoir immédiatement recours à des approches multidisciplinaires lourdes est en fait l'un des points d'achoppement majeurs de toute phase de diagnostic. Si l'on conçoit bien en effet que l'analyse des causes de non-fonctionnalité et des moyens à mettre en œuvre pour les traiter impliquent des approfondissements conséquents par champs d'expertise (techniques, sociologiques, économiques...), il paraît souhaitable de cibler au préalable ces interventions par l'identification de symptômes de non-fonctionnalité au moyen d'indicateurs simples et intégrateurs.

Un examen «naturaliste» de la mécanique globale du système de pilotage doit permettre ce préalable. L'analyse d'une représentation mise au point par l'intervenant et les acteurs (pilotes) concernés selon les grandes lignes dégagées au paragraphe précédent, permet en effet de vérifier des principes simples de cohérence et d'exhaustivité de ce système de pilotage ; on peut ainsi mettre en évidence les faiblesses de la mécanique de pilotage qui rendent difficile le déploiement des objectifs stratégiques : interface non instrumentée, pilote non opérationnel, absence de certaines com- 
munications... Le point de vue défendu est que ces faiblesses sont en fait des symptômes "intégrateurs " permettant d'aiguiller le diagnostic, à travers leur analyse, vers les causes profondes de non-fonctionnalité du système de production par rapport à la poursuite de ses objectifs stratégiques.

La recherche des causes de non-fonctionnalité doit être structurée. Trois facteurs explicatifs principaux sont repris sous des formes différentes par la plupart des auteurs ayant proposé des lectures sociologiques des systèmes de production industriels : les machines, les hommes et les institutions [7].

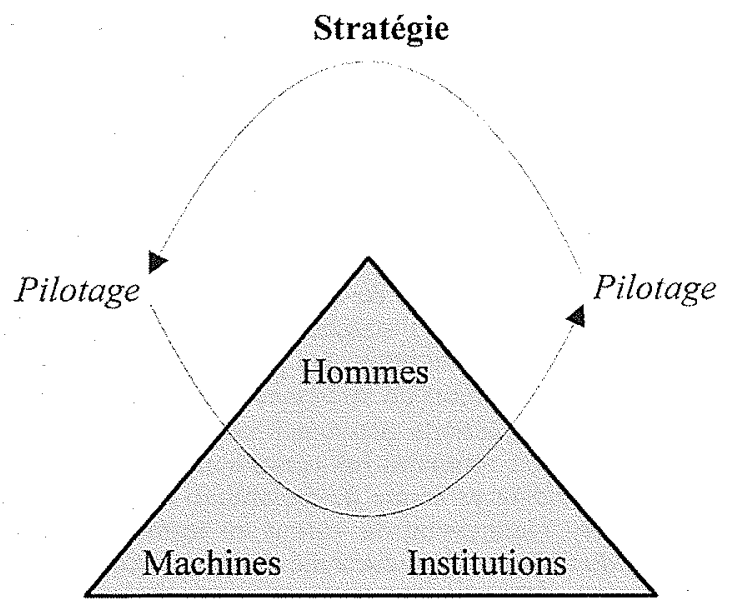

4. Stratégie, pilotage et système de production.

Dans ce cadre très simpliste, l'analyse causale est alors orientée vers la recherche d'insuffisances du système de production en matière de technologie de production (problèmes de machines: par exemple flexibilité limitée due à la nature des ouvrages hydrauliques...), en matière de décisions de pilotage (problèmes d'hommes: par exemple pilote n'ayant pas formalisé de méthode pour réguler un canal principal...) ou en matière de structure organisationnelle (problèmes d'institutions : par exemple hiérarchie d'une agence d'irrigation incompatible avec la hiérarchie de pilotage...).

La tâche est loin d'être évidente; dans un contexte fortement multi-acteur et multi-objectif, les causes de non-fonctionnalité expliquant l'état d'équilibre «dysfonctionnel » atteint par un système peuvent en effet être multiples et corrélées [8]. L'intérêt de l'analyse proposée repose en fait sur la mise en perspective et en cohérence de ces diverses causes à travers leur influence sur des faiblesses identifiées du système de pilotage.

Le traitement des causes passe enfin par la mise au point d'une nouvelle représentation du système de pilotage prenant en compte les avancées à réaliser en matière de technologie, de décisions de pilotage et d'organisation pour rendre le système de production fonctionnel par rapport à ses objectifs stratégiques. Créer les conditions d'une cohérence durable de ce système de pilotage "cible » devient en fait l'objectif explicite de l'intervention. Ce travail comprend deux volets principaux : une mise à plat et un traitement poussé «par champs d'expertise » des causes de non-fonctionnalité détectées grâce à l'analyse du système de pilotage initial, suivis de l'instrumentation (désormais durable) du nouveau système de pilotage.

\section{$\mathrm{V}$ CONCLUSIONS}

La trame d'intervention proposée se résume finalement à un parcours "d'explicitation» en trois étapes: représentation, symptômes de non-fonctionnalité, causes de non-fonctionnalité, bouclé sur la première étape qui fait office de point d'ancrage.

L'originalité de cette trame tient d'une part à l'objet du point d'ancrage, le système de pilotage, qui permet de finaliser l'intervention en tant que support concret à l'action et d'autre part au caractère nécessairement très participatif de la démarche. Tous les « acteurs pilotes » concernés doivent en effet être impliqués aux différents stades de l'intervention pour mettre à jour, discuter et valider les représentations successives du système de pilotage afin d'assurer pertinence et durabilité aux traitements envisagés sur le système de production et à la nouvelle instrumentation du pilotage qui leur est associée.

Pour reprendre un terme à la mode de la gestion en entreprise [9], le type d'intervention proposé peut finalement être considéré comme une forme de « ré-ingéniérie » du système de pilotage.

\section{BIBLIOGRAPHIE}

[1] LoRino P. (1991). - « Le Contrôle de Gestion Stratégique. La Gestion par les activités », Dunod.

[2] Shingo S. (1990). - «La Production Sans Stock», Les Editions d'Organisation.

[3] Mélèse J. (1991). - «L'analyse modulaire des systèmes », Les éditions d'organisation.

[4] Divine M. (1991). - «Parlez-vous Merise ?», Eyrolles.

[5] MélésE J. (1995), - «Approches systémiques des organisations », Les éditions d'organisation.

[6] MÉlèse P. (1995). - «Comptes et récits de la performance », Les éditions d'organisation.

[7] Rivelune C. (1991). - «Un Point de Vue d'Ingénieur sur la Gestion des Organisations », pp. 50-62 in : Annales des Mines.

[8] PERrY C. (1995). - «Function and dysfunction - a key determinant of irrigation performance and option for improvement », pp. 25-38 in water ressources development, vol. 11, number 1, march 1995.

[9] Hammer M., Champy J. (1993). - "Le reengineering *, Dunod. 